\title{
Hospital Medicine Point of Care Ultrasound Credentialing: An Example Protocol
}

\author{
Benji K. Mathews, MD ${ }^{1,2 \star}$, Michael Zwank, MD, RDMS²,3
}

${ }^{1}$ Department of Hospital Medicine, HealthPartners, University of Minnesota Medical School, Minneapolis, Minnesota; ${ }^{2}$ University of Minnesota Medical School, Minneapolis, Minnesota; ${ }^{3}$ Department of Emergency Medicine, Regions Hospital, St. Paul, Minnesota.

\begin{abstract}
Though the use of point-of-care ultrasound (POCUS) has increased over the last decade, formal hospital credentialing for POCUS may still be a challenge for hospitalists. This document details the Hospital Medicine Department Ultrasound Credentialing Policy from Regions Hospital, which is part of the HealthPartners organization in Saint Paul, Minnesota.
\end{abstract}

National organizations from internal medicine and hospital medicine (HM) have not published recommended guidelines for POCUS credentialing. Revised guidelines for POCUS have been published by the American College of Emergency Physicians, though these are not likely intended to guide hospitalists when working with credentialing committees and medical boards.

This document describes the scope of ultrasound in HM and our training, credentialing, and quality assurance program. This report is intended to be used as a guide for hospitalists as they work with their own credentialing committees and will require modification for each institution. However, the overall process described here should assist in the establishment of POCUS at various institutions. Journal of Hospital Medicine 2017;12:767-772. @ 2017 Society of Hospital Medicine
Ultrasound has been used for decades by radiology, obstetrics-gynecology, and cardiology departments within a comprehensive paradigm in which a physician enters an order, then a trained sonographer performs the study, followed by a physician evaluating and interpreting the images. ${ }^{1}$ Unlike the traditional comprehensive paradigm, point-of-care ultrasound (POCUS) is a focused study that is both performed and interpreted by the bedside provider. ${ }^{2}$ POCUS has been demonstrated to improve diagnosis and clinical management in multiple studies. ${ }^{3-15}$

The scope of practice in POCUS differs by specialty, as POCUS is done to achieve specific procedural aims (eg, direct the needle to the correct location) or answer focused questions (eg, does the patient have a distended bladder?) related to the specialty. POCUS in hospital medicine (HM) provides immediate answers, without the delay and potential risk of transportation to other hospital areas. It may be used to diagnose pleural effusion, pneumonia, hydronephrosis, heart failure, deep vein thrombosis, and many other pathologies..$^{515}$ It is important to understand that POCUS performed by HM is a limited study and is not a substitute for more complete ultrasound examinations conducted in the radiology suite or in the echocardiography lab.

Address for correspondence and reprint requests: Benji K. Mathews, MD, FACP, CLHM Department of Hospital Medicine, HealthPartners, Department of Hospital Medicine, Regions Hospital, 640 Jackson Street, Mail Stop 11109E, St. Paul, MN 55101; Telephone: 651-254-9555; Fax: 651-254-9673; E-mail: benji@umn.edu

Additional Supporting Information may be found in the online version of this article.

Received: November 1, 2016; Revised: January 17, 2017;

Accepted: February 4, 2017

2017 Society of Hospital Medicine DOI 10.12788/jhm.2809
POCUS should not be used exclusively in medical decision making, but rather in conjunction with the greater clinical context of each patient, building on established principles of diagnosis and management.

\section{DEFINITIONS}

- Credentialing: An umbrella term, which incorporates licensure, education, and certification.

- Privileging: Used to define the scope authorized for a provider by a healthcare organization based on an evaluation of the individual's credentials and performance.

- Competency: An observable ability of a provider, integrating multiple components, such as knowledge and skills. Since competencies are observable, they can be measured and assessed to ensure their acquisition.

- Certification: The process by which an association grants recognition to a provider who has met certain predetermined qualifications specified by the association. Competence is distinguished from certification, which is defined as the process by which competence is recognized by an external agency.

All of the above mechanisms work together to provide the highest quality of reliability that a practitioner is providing safe, competent care. ${ }^{16-18}$

\section{STATEMENTS FROM MAJOR SPECIALTY SOCIETIES}

Acknowledging that there are no published guidelines in the realm of HM POCUS, the development of the credentialing process at our institution is consistent with published guidelines by Emergency Medicine societies (the most established physician users of POCUS) and the American Medical Association (AMA). ${ }^{19-21}$

The use of emergency ultrasound by physicians in the emergency department is endorsed by the American College 
TABLE. Hospital Medicine Portfolio Requirements

\begin{tabular}{l}
\hline Cardiac Study (20 studies with the following images per study) $)^{22,54-76}$ \\
\hline Total: 100 images \\
1. Parasternal long axis view \\
2. Parasternal short axis view \\
3. Apical four-chamber view \\
4. Subcostal long axis view \\
5. Inferior vena cava longitudinal view
\end{tabular}

Lung/Pleural Study (5 studies with the following images per study) (3-53 $^{43}$

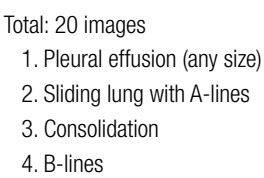

Abdominal Study (5 studies with the following images per study) ${ }^{27-34}$

Total: 20 images

1. Left kidney longitudinal view with splenorenal space

2. Right kidney longitudinal view with hepatorenal recess

3. Abdominal aorta longitudinal view

4. Bladder transverse view

Vascular Diagnostic DVT Study (3 studies with the following images per study; include right and left legs) $35-42$

Total: 24 images

1. Right common femoral vein with compression

2. Left common femoral vein with compression

3. Right common femoral vein at saphenous intake with compression

4. Left common femoral vein at saphenous intake with compression

5. Right superficial femoral vein with compression

6. Left superficial femoral vein with compression

7. Right popliteal vein with compression

8. Left popliteal vein with compression

Adapted from CHEST Critical Care Ultrasonography Program ${ }^{18,86}$

NOTE: Abbreviation: DVT, deep vein thrombosis. departments at other institutions that are attempting to create a POCUS credentialing program.

An interdisciplinary approach was created by our institution to address training, competency, and ongoing quality assurance (QA) concerns due to the increasing popularity of POCUS and variability in its use. We developed a hospital-wide POCUS committee with, among others, members from HM, emergency medicine, critical care, radiology, and cardiology, with a charter to standardize POCUS across departments. After review of the literature, ${ }^{16-18,20,21,23-74}$ baseline training requirements were established for credentialing and developing a unified delineation of privileges for hospital-wide POCUS. The data support the use of a variety of assessments to ensure a provider has developed competence (portfolio development, knowledge-based examination, skills-based assessment, ongoing QA process). The POCUS committee identified which exams could be performed at bedside for credentialed providers, delineated imaging requirements for each exam, and set up the information technology infrastructure to support ordering and reporting through electronic health records (EHR). While the POCUS committee delineated this process for all hospital providers, we will focus our discussion on the credentialing policy and procedure in HM.

\section{STEP 1: PATHWAY TO POCUS CREDENTIALING IN HM: COMPLETE MINIMAL FORMAL REQUIREMENTS}

The credentialing requirements at our institution include one of the the following basic education pathways and minimal formal training:

\section{Residency/Fellowship Based Pathway}

Completed training in an Accreditation Council for Graduate Medical Education-approved program that provided opportunities for 20 hours of POCUS training with at least 6 hours of hands-on ultrasound scanning, 5 proctored limited cardiac ultrasound cases and portfolio development.

\section{Practice Based Pathway}

Completed 20 hours of POCUS continuing medical education (CME) with at least 6 hours of hands-on ultrasound scanning and has completed 5 proctored limited cardiac ultrasound cases (as part of CME).

The majority of HM providers had little formal residency training in POCUS, so a training program needed to be developed. Our training program, modeled after the American College of Chest Physicians' CHEST certificate of completion, ${ }^{86}$ utilizes didactic training, hands-on instruction, and portfolio development that fulfills the minimal formal requirements in the practice-based pathway.

\section{STEP 2: PATHWAY TO POCUS CREDENTIALING IN HM: COMPLETE PORTFOLIO AND FINAL ASSESS- MENTS (KNOWLEDGE AND SKILLS-BASED)}

After satisfactory completion of the minimal formal training, applicants need to provide documentation of a set number of cases. To aid this requirement, our HM department developed the portfolio guidelines in the Table. These are 


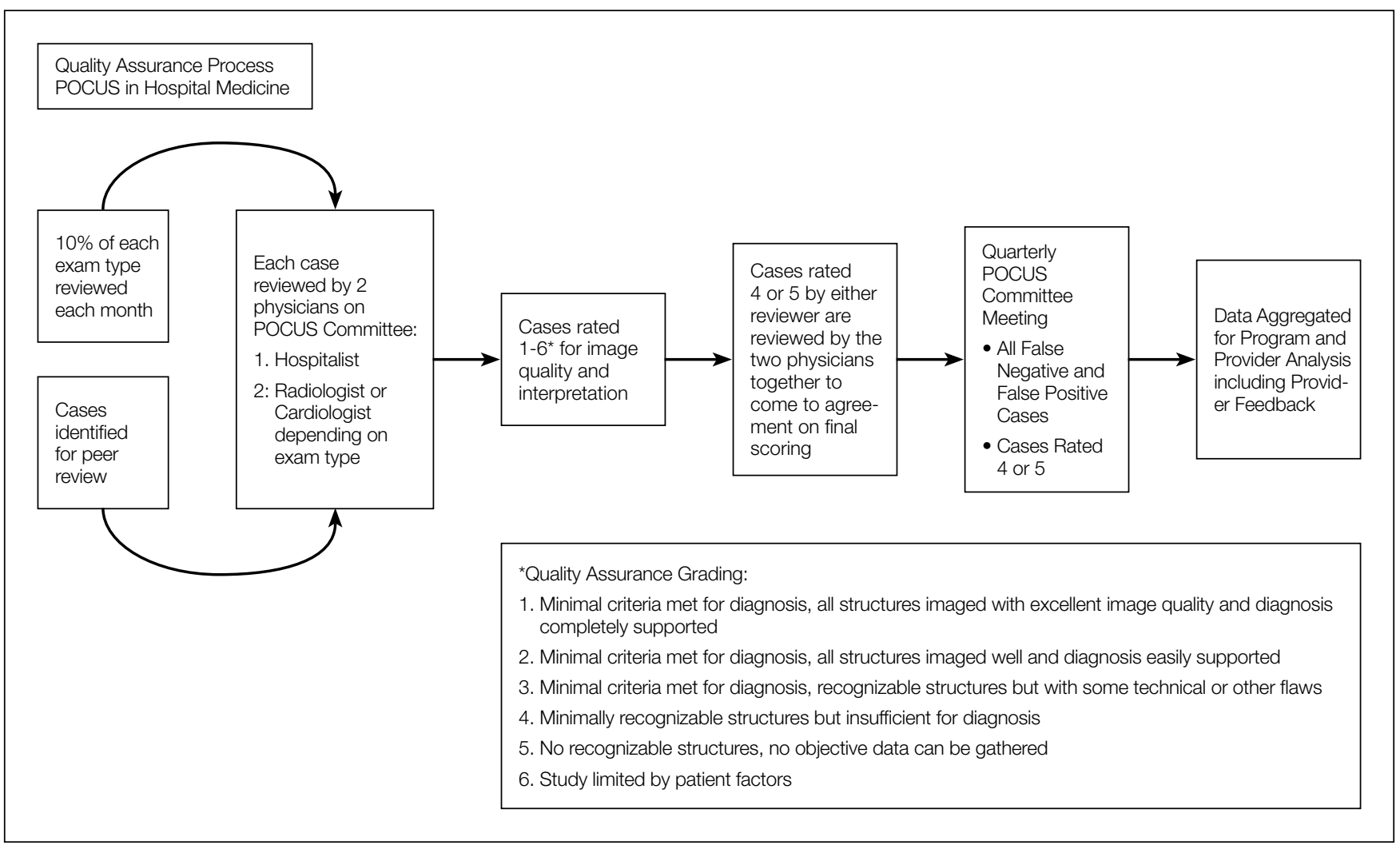

FIG. Quality Assurance process. NOTE: Abbreviation: POCUS, point-of-care ultrasound.

minimum requirements, and because of the varying training curves of learning, ${ }^{76-80} 1$ hospitalist may need to submit 300 files for review to meet the standards, while another may need to submit 500 files. Submissions are not accepted unless they yield high-quality video files with meticulous attention to gain, depth, and appropriate topographic planes. The portfolio development monitors hospitalists' progression during their deliberate practice, providing objective assessments, feedback, and mentorship. ${ }^{81,82}$

A final knowledge exam with case-based image interpretation and hands-on examination is also provided. The passing score for the written examination is $85 \%$ and was based on the Angoff methodology. ${ }^{75}$ Providers who meet these requirements are then able to apply for POCUS credentialing in HM. Providers who do not pass the final assessments are required to participate in further training before they reattempt the assessments. There is uniformity in training outcomes but diversity in training time for POCUS providers.

Candidates who complete the portfolio and satisfactorily pass the final assessments are credentialed after review by the POCUS committee. Credentialed physicians are then able to perform POCUS and to integrate the findings into patient care.

\section{MAINTENANCE OF CREDENTIALS Documentation}

After credentialing is obtained, all POCUS studies used in patient care are included in the EHR following a clearly de- fined workflow. The study is ordered through the EHR and is retrieved wirelessly on the ultrasound machine. After performing the ultrasound, all images are wirelessly transferred to the radiology Picture Archiving and Communication System server. Standardized text reports are used to distinguish focused POCUS from traditional diagnostic ultrasound studies. Documentation is optimized using electronic drop-down menus for documenting ultrasound findings in the EHR.

\section{Minimum Number of Examinations}

Maintenance of credentials will require that each hospitalist perform 10 documented ultrasounds per year for each cardiac and noncardiac application for which credentials are requested. If these numbers are not met, then all the studies performed during the previous year will be reviewed by the ultrasound committee, and providers will be provided with opportunities to meet the minimum benchmark (supervised scanning sessions).

\section{Quality Assurance}

Establishing scope of practice, developing curricula, and credentialing criteria are important steps toward assuring provider competence. . $1,17,22,74$ To be confident that providers are using POCUS appropriately, there must also be a development of standards of periodic assessment that encompass both examination performance and interpretation. The objective of a QA process is to evaluate the POCUS cases for technical competence and the interpretations for clinical accuracy, and 
to provide feedback to improve performance of providers.

QA is maintained through the interdisciplinary POCUS committee and is described in the Figure.

After initial credentialing, continued QA of HM POCUS is done for a proportion of ongoing exams $(10 \%$ as per recommendations by ACEP) to document continued competency. ${ }^{2}$ Credentialed POCUS providers perform and document their exam and interpretations. Ultrasound interpretations are reviewed by the POCUS committee (every case by 2 physicians, 1 hospitalist, and 1 radiologist or cardiologist depending on the study type) at appropriate intervals based on volume (at minimum, quarterly). A standardized review form is used to grade images and interpretations. This is the same general rubric used with the portfolio for initial credentialing. Each case is scored on a scale of 1 to 6 , with 1 representing high image quality and support for diagnosis and 6 representing studies limited by patient factors. All scores rated 4 or 5 are reviewed at the larger quarterly POCUS committee meetings. For any provider scoring a 4 or 5 , the ultrasound committee will recommend a focused professional practice evaluation as it pertains to POCUS. The committee will also make recommendations on a physician's continued privileges to the department leaders. ${ }^{83}$

\section{BILLING}

Coding, billing, and reimbursement for focused ultrasound has been supported through the AMA Physicians' Current Procedural Terminology (CPT) 2011 codes, which includes CPT code modifiers for POCUS. ${ }^{84}$ There are significant costs associated with building a HM ultrasound program, including the education of hospitalists, ultrasound equipment purchase and maintenance, as well as image archiving and QA. The development of a HM ultrasound billing program can help justify and fund these costs. ${ }^{19,85}$

To appropriately bill for POCUS, permanently retrievable images and an interpretation document need to be available for review. HM coders are instructed to only bill if both components are available. Because most insurers will not pay for 2 of the same type of study performed within a 24 -hour period, coders do not bill for ultrasounds when a comprehensive ultrasound of the same body region is performed within a 24-hour period. The workflow that we have developed, including ordering, performing, and documenting, allows for easy coding and billing.

\section{BARRIERS AND LIMITATIONS}

While POCUS has a well-established literature base in other specialties like emergency medicine, it has been a relatively recent addition to the HM specialty. As such, there exists a paucity of evidence-based medicine to support its use of POCUS in HM. While it is tempting to extrapolate from the literature of other specialties, this may not be a valid approach.

Training curves in which novice users of ultrasound become competent in specific applications are incompletely understood. Little research describes the rate of progression of learners in ultrasound towards competency. We have recently started the QA process and hope that the data will further guide feedback to the process.

Additionally, with the portfolios, the raters' expertise may not be stable (develops through experience). We aim to mitigate this by having a group of raters reviewing each file, particularly if there is a question about if a submission is of high image quality. A notable barrier that groups face is support from their leadership regarding POCUS. Our group has had support from the chief medical officer who helped mandate the development of POCUS standards.

\section{LESSONS LEARNED}

We have developed a robust collaborative HM POCUS program. We have noted challenges in motivating all providers to work through this protocol. Development of a POCUS program takes dedicated time, and without a champion, it is at risk for failing. HM departments would be advised to seek out willing collaborators at their institutions. We have seen that it is useful to partner with some experienced emergency medicine providers. Additionally, portfolio development and feedback has been key to demonstrating growth in image acquisition. Deliberate longitudinal practice with feedback and successive refinements with POCUS obtain the highest yield towards competency. We hope our QA data will provide further feedback into the credentialing policy and procedure.

\section{SUMMARY}

It is important that POCUS users work together to recognize its potential and limitations, teach current and future care providers' best practices, and create an infrastructure that maximizes quality of care while minimizing patient risk.

We are hopeful that this document will prove beneficial to other HM departments in the development of successful POCUS programs. We feel that it is important to make available to other HM departments a concise protocol that has successfully passed through the credentialing process at a large tertiary care medical system.

\section{Acknowledgements}

The authors would like to acknowledge Susan Truman, MD, for her contributions to the success of the POCUS committee at Regions Hospital. The authors would like to acknowledge Kreegan Reierson, MD, Ankit Mehta, MBBS, and Khuong Vuong, MD for their contributions to the success of POCUS within hospital medicine at HealthPartners. The authors would like to acknowledge Sandi Wewerka, MPH, for her review and input of this manuscript.

Disclosure: The authors do not have any relevant financial disclosures to report.

\section{References}

1. Soni NJ, Nilam J, Arntfield R, Kory P. Point of Care Ultrasound. Philadelphia: Elsevier; 2015.

2. Moore CL, Copel JA. Point-of-Care Ultrasonography. $N$ Engl J Med. 2011;364(8):749-757

3. Randolph AG, Cook DJ, Gonzales CA, et al. Ultrasound guidance for placement of central venous catheters: A meta-analysis of the literature. Crit Care Med. $1996 ; 24: 2053-2058$. 
4. Gordon CE, Feller-Kopman D, Balk EM, et al. Pneumothorax following thoracen tesis: A systematic review and meta-analysis. Arch Intern Med. 2010;170:332-339.

5. Soni NJ, Nilam J, Franco R, et al. Ultrasound in the diagnosis and management of pleural effusions. J Hosp Med. 2015;10(12):811-816.

6. Nazerian P, Volpicelli G, Gigli C, et al. Diagnostic performance of Wells score combined with point-of-care lung and venous ultrasound in suspected pulmonary embolism. Acad Emerg Med. 2017;24(3):270-280.

7. Chatziantoniou A, Nazerian P, Vanni S, et al. A combination of the Wells score with multiorgan ultrasound to stratify patients with suspected pulmonary embolism. Eur Respir J. 2015;46:OA493; DOI:10.1183/13993003.congress-2015. OA493.

8. Boyd JH, Sirounis D, Maizel J, Slama M. Echocardiography as a guide for fluid management. Crit Care. 2016; DOI:10.1186/s13054-016-1407-1.

9. Mantuani D, Frazee BW, Fahimi J, Nagdev A. Point-of-Care Multi-Organ Ultrasound Improves Diagnostic Accuracy in Adults Presenting to the Emergency Department with Acute Dyspnea. West J Emerg Med. 2016;17(1):46-53.

10. Glockner E, Christ M, Geier F, et al. Accuracy of Point-of-Care B-Line Lung Ultrasound in Comparison to NT-ProBNP for Screening Acute Heart Failure. Ultrasound Int Open. 2016;2(3):e90-e92.

11. Bhagra A, Tierney DM, Sekiguchi H, Soni NJ. Point-of-Care Ultrasonog raphy for Primary Care Physicians and General Internists. Mayo Clin Proc. 2016;91(12):1811-1827

12. Crisp JG, Lovato LM, and Jang TB. Compression ultrasonography of the lower extremity with portable vascular ultrasonography can accurately detect deep venous thrombosis in the emergency department. Ann Emerg Med. 2010;56:601-610.

13. Squire BT, Fox JC, and Anderson C. ABSCESS: Applied bedside sonography for convenient. Evaluation of superficial soft tissue infections. Acad Emerg Med 2005;12:601-606

14. Narasimhan M, Koenig SJ, Mayo PH. A Whole-Body Approach to Point of Care Ultrasound. Chest. 2016;150(4):772-776.

15. Oks M, Cleven KL, Cardenas-Garcia J, et al. The effect of point-of-care ultrasonography on imaging studies in the medical ICU: a comparative study. Chest. 2014;146(6):1574-1577

16. Mayo PH, Beaulieu Y, Doelken P, et al. American College of Chest Physicians/ La Société de Réanimation de Langue Française Statement on Competence in Critical Care Ultrasonography. Chest. 2009;135(4):1050-1060.

17. Frank JR, Snell LS, Ten Cate O, et al. Competency-based medical education Theory to practice. Med Teach. 2010;32:638-645.

18. The Who, What, When, and Where's of Credentialing and Privileging. The Joint Commission. http://www.jointcommission.org/assets/1/6/AHC_who_what_ when_and_where_credentialing_booklet.pdf. Accessed December 21, 2016.

19. American College of Emergency Physicians Policy Statement: Emergency Ultrasound Guidelines. 2016. https://www.acep.org/Clinical-..Practice-Management/ ACEP-Ultrasound-Guidelines/. Accessed October 26, 2016

20. Society for Academic Emergency Medicine. Ultrasound Position Statement. An nual Meeting 1996

21. American Medical Association. Privileging for ultrasound imaging. 2001; Policy H-230.960. www.ama-assn.org. Accessed July 28, 2017

22. Stein JC, Nobay F. Emergency Department Ultrasound Credentialing: a sample policy and procedure. J Emerg Med. 2009;37(2):153-159.

23. Spencer KT, Kimura BJ, Korcarz CE, Pellikka PA, Rahko PS, Siegel RJ. Focused Cardiac Ultrasound: Recommendations from the American Society of Echocardiography. J Am Soc Echocardiogr. 2013;26(6):567-581

24. Wiegers S. The Point of Care. J Am Soc Echocardiogr. 2016;29(4):19.

25. Mandavia D, Aragona J, Chan L, et al. Ultrasound training for emergency physicians-a prospective study. Acad Emerg Med. 2000;7:1008-1014.

26. American College of Radiology Practice Parameters and Technical Standards. https://www.acr.org/quality-safety/standards-guidelines. Accessed December 21, 2016.

27. Blois B. Office-based ultrasound screening for abdominal aortic aneurysm. Can Fam Physician. 2012;58(3):e172-e178

28. Rubano E, Mehta N, Caputo W, Paladino L, Sinert R. Systematic review: emergency department bedside ultrasonography for diagnosing suspected abdominal aortic aneurysm. Acad Emerg Med. 2013;20:128-138.

29. Dijos M, Pucheux Y, Lafitte M, et al. Fast track echo of abdominal aortic aneurysm using a real pocket-ultrasound device at bedside. Echocardiography. 2012;29(3):285-290

30. Cox C, MacDonald S, Henneberry R, Atkinson PR. My patient has abdominal and flank pain: Identifying renal causes. Ultrasound. 2015;23(4):242-250.

31. Gaspari R, Horst K. Emergency ultrasound and urinalysis in the evaluation of patients with flank pain. Acad Emerg Med. 2005;12:1180-1184.

32. Kartal M, Eray O, Erdogru T, et al. Prospective validation of a current algorithm including bedside US performed by emergency physicians for patients with acute flank pain suspected for renal colic. Emerg Med J. 2006;23(5):341-344.

33. Noble VE, Brown DF. Renal ultrasound. Emerg Med Clin North Am. 2004;22:641-659.

34. Surange R, Jeygopal NS, Chowdhury SD, et al. Bedside ultrasound: a useful too for the on call urologist? Int Urol Nephrol. 2001;32:591-596.

35. Pomero F, Dentali F, Borretta V, et al. Accuracy of emergency physician-performed ultrasonography in the diagnosis of deep-vein thrombosis. Thromb Haemost. 2013;109(1):137-145

36. Bernardi E, Camporese G, Buller HR, et al. Erasmus Study Group. Serial 2-Point Ultrasonography Plus D-Dimer vs Whole-Leg Color-Coded Doppler Ultrasonography for Diagnosing Suspected Symptomatic Deep Vein Thrombosis: A Randomized Controlled Trial. JAMA. 2008;300(14):1653-1659.

37. Burnside PR, Brown MD, Kline JA. Systematic Review of Emergency Physicianperformed Ultrasonography for Lower-Extremity Deep Vein Thrombosis. Acad Emerg Med. 2008;15:493-498.

38. Magazzini S, Vanni S, Toccafondi S, et al. Duplex ultrasound in the emergency department for the diagnostic management of clinically suspected deep vein thrombosis. Acad Emerg Med. 2007;14:216-220.

39. Jacoby J, Cesta M, Axelband J, Melanson S, Heller M, Reed J. Can emergency medicine residents detect acute deep venous thrombosis with a limited, two-site ultrasound examination? J Emerg Med. 2007;32:197-200.

40. Jang T, Docherty M, Aubin C, Polites G. Resident-performed compression ultrasonography for the detection of proximal deep vein thrombosis: fast and accurate. Acad Emerg Med. 2004;11:319-322.

41. Frazee BW, Snoey ER, Levitt A. Emergency Department compression ultrasound to diagnose proximal deep vein thrombosis. J Emerg Med. 2001;20:107-112.

42. Blaivas M, Lambert MJ, Harwood RA, Wood JP, Konicki J. Lower-extremity Doppler for deep venous thrombosis--can emergency physicians be accurate and fast? Acad Emerg Med. 2000;7:120-126.

43. Koenig SJ, Narasimhan M, Mayo PH. Thoracic ultrasonography for the pulmonary specialist. Chest. 2011;140(5):1332-1341.

44. Lichtenstein, DA. A bedside ultrasound sign ruling out pneumothorax in the critically ill. Lung sliding. Chest. 1995;108(5):1345-1348.

45. Lichtenstein D, Mézière G, Biderman P, Gepner A, Barré O. The comet-tail artifact. An ultrasound sign of alveolar-interstitial syndrome. Am J Respir Crit Care Med. 1997;156(5):1640-1646.

46. Copetti R, Soldati G, Copetti P. Chest sonography: a useful tool to differentiate acute cardiogenic pulmonary edema from acute respiratory distress syndrome. Cardiovasc Ultrasound. 2008;6:16.

47. Agricola E, Bove T, Oppizzi M, et al. Ultrasound comet-tail images: a marker of pulmonary edema: a comparative study with wedge pressure and extravascular lung water. Chest. 2005;127(5):1690-1695.

48. Lichtenstein DA, Meziere GA, Laqoueyte JF, Biderman P, Goldstein I, Gepner A. A-lines and B-lines: lung ultrasound as a bedside tool for predicting pulmonary artery occlusion pressure in the critically ill. Chest. 2009;136(4):1014-1020.

49. Lichtenstein DA, Lascols N, Meziere G, Gepner A. Ultrasound diagnosis of alveolar consolidation in the critically ill. Intensive Care Med. 2004;30(2):276-281.

50. Lichtenstein D, Mezière G, Seitz J. The dynamic air bronchogram. A lung ultrasound sign of alveolar consolidation ruling out atelectasis. Chest. 2009;135(6):1421-1425.

51. Lichtenstein D, Goldstein I, Mourgeon E, Cluzel P, Grenier P, Rouby JJ. Comparative diagnostic performances of auscultation, chest radiography, and lung ultrasonography in acute respiratory distress syndrome. Anesthesiology. 2004;100(1):9-15.

52. Lichtenstein D, Meziere G. Relevance of lung ultrasound in the diagnosis of acute respiratory failure: the BLUE protocol. Chest. 2008;134(1):117-125

53. Mayo P, Doelken P. Pleural ultrasonography. Clin Chest Med. 2006;27(2):215-227.

54. Galderisi M, Santoro A, Versiero M, et al. Improved cardiovascular diagnostic accuracy by pocket size imaging device in non-cardiologic outpatients: the NaUSiCa (Naples Ultrasound Stethoscope in Cardiology) study. Cardiovasc Ultrasound. 2010;8:51

55. DeCara JM, Lang RM, Koch R, Bala R, Penzotti J, Spencer KT. The use of small personal ultrasound devices by internists without formal training in echocardiography. Eur J Echocardiography. 2002;4:141-147.

56. Martin LD, Howell EE, Ziegelstein RC, Martire C, Shapiro EP, Hellmann DB. Hospitalist performance of cardiac hand-carried ultrasound after focused training. Am J Med. 2007;120:1000-1004.

57. Martin LD, Howell EE, Ziegelstein RC, et al. Hand-carried ultrasound performed by hospitalists: does it improve the cardiac physical examination? Am J Med. 2009; $122: 35-41$.

58. Perez-Avraham G, Kobal SL, Etzion O, et al. Left ventricular geometric abnormality screening in hypertensive patients using a hand-carried ultrasound device. J Clin Hypertens. 2010;12:181-186.

59. Lucas BP, Candotti C, Margeta B, et al. Diagnostic accuracy of hospitalist-performed hand-carried ultrasound echocardiography after a brief training program. $J$ Hosp Med. 2009;4:340-349.

60. Kimura BJ, Fowler SJ, Fergus TS, et al. Detection of left atrial enlargement using hand-carried ultrasound devices to screen for cardiac abnormalities. Am J Med. 2005;118:912-916.

61. Brennan JM, Blair JE, Goonewardena S, et al. A comparison by medicine resi- 
dents of physical examination versus hand-carried ultrasound for estimation of right atrial pressure. Am J Cardiol. 2007;99:1614-1616.

62. Blair JE, Brennan JM, Goonewardena SN, Shah D, Vasaiwala S, Spencer KT. Usefulness of hand-carried ultrasound to predict elevated left ventricular filling pressure. Am J Cardiol. 2009;103:246-247.

63. Stawicki SP, Braslow BM, Panebianco NL, et al. Intensivist use of hand-carried ultrasonography to measure IVC collapsibility in estimating intravascular volume status: correlations with CVP. J Am Coll Surg. 2009;209:55-61.

64. Gunst M, Ghaemmaghami V, Sperry J, et al. Accuracy of cardiac function and volume status estimates using the bedside echocardiographic assessment in trauma/ critical care. J Trauma. 2008;65:509-515.

65. Razi R, Estrada JR, Doll J, Spencer KT. Bedside hand-carried ultrasound by internal medicine residents versus traditional clinical assessment for the identification of systolic dysfunction in patients admitted with decompensated heart failure. J Am Soc Echocardiogr. 2011;24:1319-1324.

66. Croft LB, Duvall WL, Goldman ME. A pilot study of the clinical impact of hand-carried cardiac ultrasound in the medical clinic. Echocardiography. 2006;23:439-446

67. Vignon P, Dugard A, Abraham J, et al. Focused training for goal-oriented handheld echocardiography performed by noncardiologist residents in the intensive care unit. Intensive Care Med. 2007;33:1795-1799.

68. Melamed R, Sprenkle MD, Ulstad VK, Herzog CA, Leatherman JW. Assessment of left ventricular function by intensivists using hand-held echocardiography. Chest. 2009; 135:1416-1420.

69. Mark DG, Hayden GE, Ky B, et al. Hand-carried echocardiography for assessment of left ventricular filling and ejection fraction in the surgical intensive care unit. J Crit Care. 2009;24(3):470.e1-470.e7.

70. Kirkpatrick JN, Davis A, Decara JM, et al. Hand-carried cardiac ultrasound as a tool to screen for important cardiovascular disease in an underserved minority health care clinic. J Am Soc Echocardiogr. 2004;17:399-403.

71. Fedson S, Neithardt G, Thomas P, et al. Unsuspected clinically important findings detected with a small portable ultrasound device in patients admitted to a general medicine service. J Am Soc Echocardiogr. 2003;16:901-905.

72. Ghani SN, Kirkpatrick JN, Spencer, KT, et al. Rapid assessment of left ventricular systolic function in a pacemaker clinic using a hand-carried ultrasound device. J Interv Card Electrophysiol. 2006;16:39-43.

73. Kirkpatrick JN, Ghani SN, Spencer KT. Hand carried echocardiography screening for LV systolic dysfunction in a pulmonary function laboratory. Eur J Echocardiogr. 2008;9:381-383

74. Alexander JH, Peterson ED, Chen AY, Harding TM, Adams DB, Kisslo JA Jr. Feasibility of point-of-care echocardiography by internal medicine house staff. Am Heart J. 2004;147:476-481.

75. Angoff WH. Scales, norms and equivalent Scores. Washington, DC: American Council on Education; 1971.

76. Hellmann DB, Whiting-O'Keefe Q, Shapiro EP, Martin LD, Martire C, Ziegelstein RC. The rate at which residents learn to use hand-held echocardiography at the bedside. Am J Med. 2005;118:1010-1018.

77. Kimura BJ, Amundson SA, Phan JN, Agan DL, Shaw DJ. Observations during development of an internal medicine residency training program in cardiovascular limited ultrasound examination. J Hosp Med. 2012;7:537-542.

78. Akhtar S, Theodoro D, Gaspari R, et al. Resident training in emergency ultrasound: consensus recommendations from the 2008 Council of Emergency Medicine Residency Directors Conference. Acad Emerg Med. 2009;16(s2):S32-S36.

79. Ma OJ, Gaddis G, Norvell JG, Subramanian S. How fast is the focused assessment with sonography for trauma examination learning curve? Emerg Med Australas. 2008;20(1):32-37

80. Gaspari RJ, Dickman E, Blehar D. Learning curve of bedside ultrasound of the gallbladder. J Emerg Med. 2009;37(1):51-56. DOI:10.1016/j.jemermed.2007.10.070.

81. Ericsson KA, Lehmann AC. Expert and exceptional performance: Evidence of maximal adaptation to task constraints. Ann Rev Psychol. 1996;47:273-305.

82. Ericcson KA, Krampe RT, Tesch-Romer C. The role of deliberate practice in the acquisition of expert performance. Psychol Rev. 1993;100:363-406.

83. OPPE and FPPE: Tools to help make privileging decisions. The Joint Commission. 2013. http://www.jointcommission.org/jc_physician_blog/oppe_fppe_tools_privileging_decisions/ Accessed October 26, 2016.

84. American Medical Association. Physicians' Current Procedural Terminology (CPT) 2011. American Medical Association, Chicago; 2011.

85. Moore CL, Gregg S, Lambert M. Performance, training, quality assurance, and reimbursement of emergency physician-performed ultrasonography at academic medical centers. J Ultrasound Med. 2004;23(4):459-466.

86. Critical Care Ultrasonography Certificate of Completion Program. CHEST. American College of Chest Physicians. http://www.chestnet.org/Education/Advanced-Clinical-Training/Certificate-of-Completion-Program/Critical-Care-Ultrasonography. Accessed July 28, 2017. 